\title{
Correlación entre Índice de Masa Corporal y Circunferencia de Cintura en una Muestra de Niños, Adolescentes y Adultos con Discapacidad de Temuco, Chile
}

\author{
Correlation between BMI and Waist Circumference in a Sample \\ of Children, Adolescents and Adults with Disabilities in Temuco - Chile
}

"Rodrigo Ojeda Nahuelcura \& **Mauricio Cresp Barría

OJEDA, N. R. \& CRESP, B. M. Correlación entre Índice de masa corporal y circunferencia de cintura en una muestra de niños, adolescentes y adultos con discapacidad de Temuco, Chile. Int. J. Morphol., 29(4):1326-1330, 2011.

RESUMEN: La obesidad es uno de los problemas de salud pública más importantes, por lo que su detección en forma temprana facilita la aplicación de estrategias preventivas. Según la Organización Mundial de la Salud, el Índice de masa corporal (IMC), es un instrumento válido para determinar el estado nutricional de la población, afirmación no compartida por especialistas del alto rendimiento. Se realiza este estudio para correlacionar el IMC con la circunferencia de cintura (CC), indicador que permite predecir con mayor exactitud la obesidad central o abdominal. El segundo objetivo de esta investigación es describir la prevalencia del sobrepeso y la obesidad en personas con discapacidad. Se evaluó a 188 niños, adolescentes y adultos jóvenes de ambos sexos, 123 hombres (65,4\%) y 65 mujeres (34,6\%), entre 3 y 25 años de edad, alumnos de escuelas especiales de la ciudad de Temuco. Para obtener el IMC y la CC se utilizó el método descrito por la OMS, efectuándose la evaluación y clasificación de acuerdo a los puntos de corte por edad de la OMS. Existe un alto grado de correlación entre IMC y CC (0,846). El 49\% del total de la muestra presentan sobrepeso u obesidad. El 43,9\% de los hombres y el 58,4\% de las mujeres presentan obesidad o sobrepeso. En cuanto a la circunferencia de cintura, el 54,3\% de la muestra presenta riesgo relativo. En los hombres el 46,3\% y en las mujeres el 69,2\% presenta riesgo relativo. El 71,88\% de las personas con síndrome de Down presentan sobrepeso y obesidad, seguidos por las personas con TGD y discapacidad intelectual con el $50 \%$ y el $46 \%$ respectivamente. El mayor riesgo relativo según la circunferencia de cintura, lo presentan las personas con discapacidad intelectual con el 58,6\% seguido por los que presentan Síndrome de Down con el 56,3\%.

PALABRAS CLAVE: Índice de masa corporal; Circunferencia de cintura; Obesidad; Sobrepeso; Personas con discapacidad; Niños; Adolescentes; Adultos jóvenes.

\section{INTRODUCCIÓN}

Actualmente uno de los problemas de salud pública más importantes es la obesidad infantil, tanto en países desarrollados como en aquellos en vías de desarrollo (Silva $e t$ al., 2008), y nuestro país Chile no es la excepción. La multiplicidad de los factores involucrados en la génesis de la obesidad y la presencia de trastornos metabólicos asociados, dificultan su prevención y tratamiento (Burrows et al., 2001). A lo anterior se agregan los altos índices de sedentarismo en la población chilena, que de acuerdo a la encuesta nacional de salud equivale al 89,2\% (MINSAL, 2010). El impacto del sedentarismo en la población no deja de ser importante, considerando que es una de las condicionantes que contribuye a que por medio del bajo gasto energético de un individuo, en conjunto con ingestas alimentarias normales o elevadas, se provoque un desbalance energético que se puede traducir en alteraciones del estado de salud, como la malnutrición por exceso. Todo esto recrudece el impacto en la comunidad, originado de la pandemia en que se ha convertido la obesidad (Buhring et al., 2009), y el denominado síndrome metabólico , que comprende un conjunto de factores de riesgo tales como; Dislipidemias, tensión arterial elevada, resistencia a la insulina, obesidad abdominal que incrementan el riesgo de enfermedad cardiovascular (Kang et al., 2008), constituyendo un estado de alto riesgo de morbilidad y mortalidad por enfermedades del corazón y diabetes, y las personas sedentarias son particularmente susceptibles a padecerlo.

* Académico de Pedagogía en Educación Física. Subdirector del Centro de Desarrollo e Innovación a la Docencia (CeDID), Universidad Católica de Temuco, Temuco, Chile.

** Director de la Carrera de Pedagogía en Educación Física. Facultad de Educación. Universidad Católica de Temuco, Temuco, Chile. 
Aunque a nivel nacional e internacional se han desarrollado diversas investigaciones respecto a la valoración antropométrica de distintas poblaciones y rangos etáreos, es importante continuar desarrollando estudios que permitan evidenciar lo que sucede con una población generalmente no considerada y que permita desarrollar conocimiento relevante para la toma de decisiones frente a las políticas de salud o educación referidas al sedentarismo, el sobrepeso y la obesidad de las personas con discapacidad. Ya en el siglo XXI la Organización Mundial de la Salud ha hecho pública una nueva versión de la CIDDM, que ha pasado a denominarse Clasificación Internacional del Funcionamiento, de la Discapacidad y de la Salud (CIF). Esta nueva versión fue aprobada por la Asamblea Mundial de la Salud, en su resolución 54.21, de 22 de mayo de 2001, bajo el título: Clasificación Internacional del Funcionamiento, de la Discapacidad y de la Salud (que utilizará CIF como nuevo acrónimo). Con esta nueva versión se pretende dar un nuevo enfoque al concepto de discapacidad, en busca de una visión integradora, más compleja e interaccional. Mientras que el punto de vista de la discapacidad en la CIDDM era calificado como lineal (donde una enfermedad o trastorno da lugar a una deficiencia, que dará lugar a su vez a una discapacidad, que determinará la existencia de una minusvalía en el sujeto), se busca ahora un enfoque biopsicosocial, esto es, en el que se tengan en cuenta tres niveles a los que referir la valoración: nivel biológico (las condiciones de salud), nivel de la persona (factores personales como la edad, el sexo, el nivel de educación) y nivel social (factores del medio social y físico). La discapacidad, para la Organización Mundial de la Salud, deja de ser algo en sí, para ser la designación del cruce de numerosas variables (Sanjosé, 2007) Para efecto de este artículo se ha subdivido en relación a los cuadros diagnósticos más recurrentes en las escuelas especiales: Síndrome de Down, discapacidad intelectual, discapacidades motoras, parálisis cerebral y trastorno general del desarrollo (TGD).

En cuanto a la identificación de la obesidad en los niños, se ha discutido un tema importante ¿Cuál es el método más exacto para clasificarla? Según la Organización Mundial de la Salud (WHO, 2009), el índice de masa corporal (IMC), obtenido por medio de la división del peso corporal, en kilos, por la estatura en metros cuadrados, es una medida antropométrica ampliamente utilizada en la identificación de sobrepeso en niños, adolescentes y adultos. Sin embargo poco contribuye a la determinación del tejido adiposo, ya que no distingue la masa magra de la masa grasa, y por lo tanto no representa la distribución de la grasa corporal (Benjumea et al., 2008). Los puntos de corte propuestos por la Organización Mundial de la Salud (WHO) se utilizaron como criterio de diagnóstico del estado nutricional.
De la misma forma, la circunferencia de cintura (CC), definida por medio de la medida de menor circunferencia entre la cresta iliaca y el reborde costa, y es particularmente mejor predictor de la obesidad visceral, una enfermedad que representa alto riesgo de desarrollar enfermedades crónicas no trasmisibles como la diabetes mellitus tipo 2 y enfermedades (Benjumea et al.). La CC se analizó desde el punto de corte propuesto por la Organización Mundial salud. Las mujeres con valores por encima de $80 \mathrm{~cm}$ y los hombres con valores superiores a $94 \mathrm{~cm}$ fueron clasificados como una acumulación de grasa abdominal, considerada un riesgo asociado con el desarrollo de enfermedades relacionadas con la obesidad.

Las investigaciones a nivel nacional que relacionan el índice de masa corporal y circunferencia de la cintura en niños y adolescentes todavía se consideran escasas en especial en muestras de personas con discapacidad.

Por lo tanto, este estudio tiene por objeto establecer una correlación entre el IMC y la CC, para caracterizar la muestra según edad, sexo y discapacidad. Además de describir la prevalencia de sobrepeso, la obesidad y la obesidad en los niños, adolescentes y adultos de las escuelas especiales Nielol y Liwen de la ciudad de Temuco.

\section{SUJETOS Y MÉTODO}

Se evaluó la circunferencia de cintura e Índice de Masa Corporal en 188 niños y jóvenes (123 hombres y 65 mujeres), con edades entre 4 y 25 años, alumnos de escuelas especiales urbanas de la ciudad de Temuco de la IX Región, Chile. La distribución de los sujetos según diagnóstico se encuentran en la Tabla I.

Los sujetos fueron evaluados en sus colegios y los datos registrados en una ficha excel. Los instrumentos de medición utilizados fueron una balanza electrónica Tanita Modelo, una balanza de piso marca Detecto Modelo 339con tallímetro y una cinta métrica. Para el análisis de los datos, se utilizó el programa SPSS 17.0.

Tabla I. Distribución de los sujetos según diagnóstico.

\begin{tabular}{lcc}
\hline Diagnóstico & $\mathrm{n}$ & $\%$ \\
\hline Síndrome de Down & 32 & 17 \\
Discapacidad Intelectual & 99 & 52,7 \\
Retraso Psicomotor & 34 & 18,1 \\
Parálisis cerebral & 17 & 9 \\
TGD & 6 & 3,2 \\
\hline Total & 188 & 100 \\
\hline
\end{tabular}


Se utilizó la prueba de Komolgorov-Smirnov (KS) para evaluar la normalidad del conjunto de datos analizados. Teniendo en cuenta los valores presentados por la prueba estadística, se constató que todas las variables evidenciaron una distribución normal. Se utilizó estadística descriptiva, compuesta por media y desviación estándar. De igual forma se aplicó el proceso estadístico R de Pearson para determinar correlación entre variables de IMC y CC.

\section{RESULTADOS}

Los resultados de las variables entre el índice de masa corporal y la circunferencia de cintura en una muestra de niños, adolescentes y adultos con discapacidad de la ciudad de Temuco, Chile se encuentran expresados en las Tablas II a VII

Tabla II. Valores de centralización de edad, peso y estatura de una muestra de niños, adolescente y adultos de ambos sexos de la ciudad de Temuco, Chile. $\mathrm{n}=188$.

\begin{tabular}{lcccccc}
\hline & $\begin{array}{c}\text { Promedio } \\
\text { general }\end{array}$ & DS & $\begin{array}{c}\text { Promedio } \\
\text { hombres }\end{array}$ & DS & Promedio mujeres & DS \\
\hline Edad & 13,22 & 5,583 & 13,09 & 5,654 & 13,46 & 5,480 \\
Peso & 45,01 & 20,711 & 45,08 & 22,115 & 44,86 & 17,918 \\
Talla & 1,41 &, 214 & 1,43 &, 221 & 1,37 &, 196 \\
\hline
\end{tabular}

Tabla III. Clasificación nutricional según IMC.

\begin{tabular}{lcccccc}
\hline Rango & & Total & \multicolumn{2}{c}{ Hombres } & \multicolumn{2}{c}{ Mujeres } \\
\hline & $\mathrm{n}$ & $\%$ & $\mathrm{n}$ & $\%$ & $\mathrm{n}$ & $\%$ \\
Bajo peso & 24 & 12,8 & 19 & 15,4 & 5 & 7,7 \\
Normal & 72 & 38,3 & 50 & 40,7 & 22 & 33,8 \\
Sobre peso & 46 & 24,5 & 30 & 24,4 & 16 & 24,6 \\
Obesidad & 46 & 24,5 & 24 & 19,5 & 22 & 33,8 \\
\hline Total & 188 & 100 & 123 & 100 & 65 & 100 \\
\hline
\end{tabular}

Tabla IV. Riesgo relativo de acuerdo a circunferencia de cintura.

\begin{tabular}{lcccccc}
\hline & \multicolumn{2}{c}{ Total } & \multicolumn{2}{c}{ Hombres } & \multicolumn{2}{c}{ Mujeres } \\
\hline Normal & $\mathrm{n}$ & $\%$ & $\mathrm{n}$ & $\%$ & $\mathrm{n}$ & $\%$ \\
Riesgo elevado & 86 & 45,7 & 66 & 53,7 & 20 & 30,8 \\
\hline Total & 102 & 54,3 & 57 & 46,3 & 45 & 69,2 \\
\hline
\end{tabular}

Tabla V. Clasificación nutricional según IMC y discapacidad.

\begin{tabular}{lcccccccccc}
\hline & \multicolumn{1}{c}{ Síndrome de Down } & \multicolumn{1}{c}{ Discapacidad intelectual } & Discapacidad motora & \multicolumn{2}{c}{ Parálisis cerebral } & \multicolumn{2}{c}{ TGD } \\
\hline & $\mathrm{n}$ & $\%$ & $\mathrm{n}$ & $\%$ & $\mathrm{n}$ & $\%$ & $\mathrm{n}$ & $\%$ & $\mathrm{n}$ & $\%$ \\
\hline Bajo peso & 0 & 0 & 10 & 10,10 & 8 & 23,53 & 6 & 35,29 & 0 & 0 \\
Normal & 9 & 28,13 & 43 & 43,43 & 12 & 35,29 & 5 & 29,41 & 3 & 50,00 \\
sobre peso & 13 & 40,63 & 23 & 23,23 & 6 & 17,65 & 2 & 11,76 & 2 & 33,33 \\
Obesidad & 10 & 31,25 & 23 & 23,23 & 8 & 23,53 & 4 & 23,53 & 1 & 16,67 \\
\hline Total & 32 & 100,00 & 99 & 100,00 & 34 & 100,00 & 17 & 100,00 & 6 & 100,00 \\
\hline
\end{tabular}

Tabla VI. Riesgo relativo según diagnóstico y circunferencia de cintura.

\begin{tabular}{lcccccccccc}
\hline & \multicolumn{2}{c}{$\begin{array}{c}\text { Síndrome de } \\
\text { Down }\end{array}$} & \multicolumn{2}{c}{$\begin{array}{c}\text { Discapacidad } \\
\text { intelectual }\end{array}$} & \multicolumn{2}{c}{$\begin{array}{c}\text { Discapacidad } \\
\text { motora }\end{array}$} & \multicolumn{3}{c}{$\begin{array}{c}\text { Parálisis } \\
\text { cerebral }\end{array}$} & TGD \\
\hline Normal & $\mathrm{n}$ & $\%$ & $\mathrm{n}$ & $\%$ & $\mathrm{n}$ & $\%$ & $\mathrm{n}$ & $\%$ & $\mathrm{n}$ & $\%$ \\
Riesgo elevado & 14 & 43,8 & 41 & 41,4 & 17 & 50,0 & 11 & 64,7 & 3 & 50,0 \\
\hline Total & 18 & 56,3 & 58 & 58,6 & 17 & 50,0 & 6 & 35,3 & 3 & 50,0 \\
\hline
\end{tabular}


OJEDA, N. R. \& CRESP, B. M. Correlación entre Índice de masa corporal y circunferencia de cintura en una muestra de niños, adolescentes y adultos con discapacidad de Temuco, Chile. Int. J. Morphol., 29(4):1326-1330, 2011.

Tabla VIII. Correlación entre IMC y CC.

\begin{tabular}{llll}
\hline \multicolumn{2}{l}{ Correlations } & & \\
\hline \multirow{4}{*}{ IMC } & Pearson Correlation & 1 & Cintura \\
& Sig. (2-tailed) & &, $846^{* *}$ \\
& $\mathrm{~N}$ & 188 &, 000 \\
\multirow{4}{*}{ Cintura } & Pearson Correlation &, $846^{* *}$ & 188 \\
& Sig. (2-tailed) &, 000 & 1 \\
& $\mathrm{~N}$ & 188 & 188 \\
\hline **. Correlation is significant at the 0.01 level (2-tailed). & \\
\hline
\end{tabular}

\section{DISCUSIÓN}

Diversas investigaciones, principalmente las relacionadas con el alto rendimiento, plantean que el IMC, no es un buen predictor antropométrico debido a la imposibilidad de discriminar entre masa magra y masa grasa, no obstante es una evaluación que sigue siendo válida en el ámbito de la salud para poblaciones con rangos de normalidad (WHO). La presente investigación confirmó que existe un alto grado de correlación entre IMC y la CC. Por lo tanto, se sugiere el uso de ambas medidas antropométricas para la determinación de la distribución de la grasa en niños permitiendo la detección temprana de problemas asociados a la obesidad como riesgo cardiovascular, hipertensión, resistencia a la insulina, la dislipidemia y síndrome metabólico (Guimaraes et al., 2008; Peixoto et al., 2006).

Por otra parte se puede apreciar que el riesgo de sobrepeso y obesidad es habitualmente mucho mayor en los niños, adolescentes y adultos con discapacidad que en la población general. Una de las razones para este aumento de la vulnerabilidad a la obesidad es el hecho de que las personas con discapacidad se enfrentan a importantes obstáculos estructurales a la actividad física (WHO).

En la investigación se evidencian diferencias entre los distintos grupos, especialmente debemos mencionar que los estudiantes que presentan síndrome de Down quienes son los que muestran mayores niveles de sobrepeso y obesidad en relación al IMC y se encuentran en segundo lugar de acuerdo a la circunferencia de cintura, esto tiene relación con la predisposición genética que provoca niveles más bajos de secreción de leptina, factores fisiológicos como la hipotonía muscular o la disfunción del tiroides que acompañan al Sìndrome de Down (Proto et al., 2007; Thiel \& Fowkes, 2007; Winnick, 1995), en segundo lugar encontramos a los estudiantes con discapacidad intelectual que concentran por una parte altos índices de sobrepeso y obesidad, lo que de acuerdo a algunos autores es consecuencia de un metabolismo energético alterado (Veugelers et al., 2006), también este grupo es el que presenta mayor número de estudiantes con bajo peso (Stevenson et al., 2006), antecedente que concuerda con lo resultados encontrados en la presente investigación, en relación a que estas personas presentarían principalmente un mayor riesgo de desnutrición.

Por lo tanto una consideración importante en el desarrollo de estrategias para prevenir la obesidad infantil, tiene relación con garantizar la participación de las personas con discapacidad, estimulando y fomentando las actividades adaptadas en áreas tales como la educación física, el deporte, la recreación, la danza y las artes creativas, la nutrición, la medicina y la rehabilitación, lo que a su vez requiere formación adecuada de los profesionales que trabajan niños con discapacidad.

OJEDA, N. R. \& CRESP, B. M. Correlation between BMI and waist circumference in a sample of children, adolescents and adults with disabilities in Temuco - Chile. Int. J. Morphol., 29(4):13261330, 2011.

SUMMARY: Obesity is one of the most important public health issues and its early detection facilitates the implementation of preventive strategies. According to the World Health Organization, the Body Mass Index (BMI) is a valid tool for measuring the nutritional state of the population; however, high performance specialists do not share this assertion. The focus of this study is to correlate BMI with waist circumference (WC). The latter is an indicator that provides a more accurate prediction of the central or abdominal obesity. The second objective of this research is to describe the prevalence of overweight and obesity in people with disabilities. A number of 188 children, adolescents and young adults of both sexes were assessed, 123 men (65.4\%) and 65 women $(34.6 \%)$ between the ages of three and twenty-five. All of them were students who attended special schools in Temuco 
city. BMI and the WC data were gathered using the method described by WHO's; therefore, the assessment and classification were carried out according to the WHO's cutoff points. There is a high degree of correlation between BMI and WC (0.846). A 49\% of the sample was either overweight or obese. A $43.9 \%$ of men and $58.4 \%$ of women were either obese or overweight. As for waist circumference, $54.3 \%$ of the sample presented a relative risk. In men, $46.3 \%$ and a $69.2 \%$ in women presented a relative risk. The $71.88 \%$ of people with Down syndrome were overweight or obese, followed by people with intellectual disabilities and PDD with a $50 \%$ and $46 \%$, respectively. The higher relative risk, according to waist circumference, was in people with intellectual disabilities with a $58.6 \%$, followed by people with Down syndrome $56.3 \%$.

KEY WORDS: Obesity; Overweight; Body mass index; Waist circumference; People with disabilities; Child; Adolescent; Young adult.

\section{REFERENCIAS BIBLIOGRÁFICAS}

Benjumea, M.; Molina, D.; Arbeláez, P. \& Agudelo, L. Circunferencia de la cintura en niños y escolares manizaleños de 1 a 16 años. Rev. Colomb. Cardiol., 15(1):23-4, 2008.

Buhring, B. K.; Oliva, M. P. \& Bravo, C. C. Determinación no experimental de la conducta sedentaria en escolares. Rev. Chil. Nutr., 36(1):23-30, 2009.

Burrows, A. R.; Gattas, Z. V.; Leiva, B. L.; Barrera, A. G. \& Buegueño, A. M. Características biológicas, familiares y metabólicas de la obesidad infantil y juvenil. Rev. Med. Chile, 129(10):1155-62, 2001.

Guimaraes, I.; Almeida, A.; Santos, A. \& Barbosa, D. Pressão arterial: efeito do índice de massa corporal e da circunferência abdominal em adolescentes. Arq. Bras. Cardiol., 90(6):42632, 2008.

Kang, Y. H.; Min, H. G.; Kim, I. J.; Kim, Y. K. \& Son, S. M. Comparison of alanine aminotransferase, white blood cell count and uric acid in their association with metabolic syndrome: A study of corean adults. Endocr. J., 55(6):1093-102, 2008.

MINSAL. Encuesta Nacional de Salud Informe Final, Ministerio de Salud Santiago de Chile, 2010. Disponible en: www.epi.minsal.cl/epi/html/invest/ENS/ENS.htm

Peixoto, M.; Benicio, M.; Latorre, M. \& Jardim, P. Circunferência da cintura e índice de massa corporal como preditores da hipertensão arterial. Arq. Bras. Cardiol., 87(4):462-70, 2006.

Proto, C.; Romualdi, D.; Cento, R. M.; Romano, C.; Campagna, G. \& Lanzone, A. Free and total leptin serum levels and soluble leptin receptors levels in two models of genetic obesity: the Prader-Willi and the Down syndromes. Metabolism, 56(8):1076-80, 2007.
Sanjosé, A. El primer tratado de derechos humanos del siglo XXI: La convención de los derechos de las personas con discapacidad. Rev. Electrón. Estud. Int., 2007. Disponible en: http://www.reei.org/index.php/revista/num13/archivos/ SanjoseGil(reei13).pdf

Silva, M. H.; Collipal, L. E.; Martínez, C. \& Torres, I. Análisis del IMC y Somatotipo en una Muestra de Adolescentes con Sobrepeso y Obesidad en Temuco-Chile. Int. J. Morphol., 26(3):707-11, 2008.

Stevenson, R. D.; Conaway, M.; Chumlea, W. C.; Rosenbaum, P.; Fung, E. B.; Henderson, R. C.; Worley, G.; Liptak, G.; O'Donnell, M.; Samson-Fang, L.; Stallings, V. A. \& North American Growth in Cerebral Palsy Study. Growth and health in children with moderate-to severe cerebral palsy. Pediatrics, 118(3):1010-8, 2006.

Thiel, R. \& Fowkes, S. W. Down syndrome and thyroid dysfunction: should nutritional support be the first-line treatment? Med. Hypotheses, 69(4):809-15, 2007.

Veugelers, R.; Penning, C.; van Gulik, M. E.; Tibboel, D. \& Evenhuis, H. Feasibility of bioelectrical impedance analysis in children with a severe generalized cerebral palsy. Nutrition, 22(1):16-22, 2006.

WHO. Population-based prevention strategies for childhood obesity. Report of a WHO forum and technical meeting, Geneva, 15-17 December, 2009. Disponible en: http:// pe.skola.edu.mt/wp-content/uploads/2010/05/OMSPopulation-Based-Prevention-Strategies-for-ChildhoodObesity.pdf

Winnick, J. Adapted Physical Education and Sport. Champaign, Illinois. Human Kinetics, 1995.

Dirección para Correspondencia:

Mg. Rodrigo Ojeda Nahuelcura

Facultad de Educación

Universidad Católica de Temuco

Casilla 15-D Temuco

CHILE

Email: rojeda@uct.cl rojeda_30@hotmail.com

Recibido : 08-08-2011

Aceptado: 22-09-2011 